\title{
Proyecto Musiclang: Aptitud musical, fluidez lectora $y$ percepción intercultural de estudiantes universitarios europeos ${ }^{1}$
}

Musiclang project: Musical aptitude, reading fluency and intercultural literacy of European university students

\author{
Esther Cores Bilbao \\ Universidad de Huelva \\ esther.cores.edu@juntadeandalucia.es
}

\section{Informe}

El proyecto Musiclang (“Aptitud musical, fluidez lectora y percepción intercultural de estudiantes universitarios europeos” FFI2016-75452-R 2016-2020), financiado por el Ministerio español de Economía y Competitividad bajo el epígrafe "reto 6: Cambios e innovaciones sociales”, y liderado por la catedrática M. Carmen Fonseca-Mora, es el marco para la colaboración de una docena de universidades europeas. Su equipo investigador, nutrido por expertos de diferentes campos disciplinares como la lingüística aplicada, la psicología, la neuropsicología o la musicología, desarrolla su labor y producción científicas en alemán, español, francés, inglés y neerlandés.

El germen de la idea para el proyecto Musiclang nace fundamentalmente de los estudios del Centro de Neurociencias cognitivas de la Universidad de Marsella dirigido por Mireille Besson y de datos obtenidos en investigaciones de la Universidad de Huelva. Por un lado, los estudios de Besson y de su equipo se centran en los elementos que comparten música y lenguaje (Moreno, Marques, Santos, Castro \& Besson, 2008), así como en el entrenamiento musical como técnica de mejora del proceso lector en lengua materna de alumnado con dislexia (Habib, Lardy, Desiles, Commeiras, Chobert, \& Besson, 2016).

Por otro lado, con respecto a la relación entre música y lenguaje en lenguas extranjeras, los resultados de la tesis doctoral de Toscano-Fuentes (2010) apuntan a que la implementación de materiales didácticos con contenidos musicales en el aula de inglés afecta principalmente a las habilidades lectoras del alumnado de sexto de primaria, mientras que los resultados de

${ }^{1}$ Recibido: 02/01/2020 Evaluado: 15/01/2020 Aceptado: 16/01/2020 
Gómez-Domínguez, Fonseca-Mora y Machancoses (2019) confirman la influencia de la aptitud musical de niños de segundo de primaria en su aprendizaje de la lectura tanto en su lengua materna como en inglés como lengua extranjera.

Partiendo de la definición de aptitud musical como capacidad de estructuración auditiva (Karma, 2007), en la que el oyente no se limita a recibir la información sonora, sino que discrimina las diferentes estructuras contenidas en una secuencia de sonidos, el equipo de Musiclang estudia la influencia de esta habilidad en el aprendizaje de lenguas extranjeras, así como la incidencia del entrenamiento musical en las destrezas receptivas que permiten una mejor comprensión del texto escrito.

Las temáticas de investigación en las que se vertebra Musiclang son, fundamentalmente, tres: 1) los factores intervinientes en el proceso lector de alumnado adulto de lenguas extranjeras, 2) el nivel de competencia intercultural existente en dicha población, y las diferentes identidades (locales, regionales, nacionales y supranacionales) que se atribuyen para su autodefinición, así como los contextos en los que se activan o se solapan cada una de esas identidades, y 3) el desarrollo de recursos didácticos audiovisuales que puedan favorecer la conciencia fonológica, la adquisición de vocabulario y la fluidez lectora en lenguas extranjeras, además de lograr el bienestar emocional y ampliar el horizonte multicultural de quienes las estudian.

Para la primera línea temática, el equipo investigador ha estudiado elementos como la fluidez y automaticidad lectoras, la prosodia, la memoria de trabajo, la velocidad de procesamiento y la comprensión verbal, para establecer el perfil lector del alumnado y vincularlo con su aptitud musical (Fonseca-Mora \& Fernández-Corbacho, 2017). La extensa producción de artículos científicos, libros y capítulos de libros derivados de este eje temático abordan el proceso lector en toda su complejidad, realizando también aportaciones en el ámbito de la enseñanza bilingüe (Foncubierta, Machancoses \& Fonseca-Mora, 2018), caracterizada por el aprendizaje integrado de contenidos y lengua extranjera, y ayudando a comprender la interrelación de los mecanismos cognitivos, afectivos y socioculturales que intervienen en la lectura en lenguas distintas a la materna (Foncubierta \& Fonseca-Mora, 2018; Legaz, 2018).

Para el análisis de la competencia intercultural, los trabajos de Musiclang han partido de las atribuciones identitarias y de la autopercepción como ciudadanos europeos de la población estudiantil universitaria, realizado una revisión de las contribuciones científicas de corte cualitativo publicadas desde los años noventa (Cores-Bilbao, Méndez-García \& FonsecaMora, 2020). Los resultados del proyecto sobre esta temática abarcan, además, artículos en los que se examina la efectividad de los programas educativos europeos para el fomento de los valores cívicos comunitarios, pluriculturales y democráticos, y en los que se plantean propuestas para el diseño de futuras políticas formativas para la ciudadanía (Méndez García, Cores-Bilbao \& Moreno Gámez, 2019).

Dentro de las competencias cívicas y democráticas, el proyecto ha dedicado especial atención al papel de la mediación lingüística, ya que se trata de una actividad de la lengua profusamente tratada en la actualización al Marco Común Europeo de Referencia para la enseñanza de lenguas que presenta el potencial de desarrollar las competencias interpersonales y de colaboración de los estudiantes, promoviendo su papel como miembros 
activos de una sociedad más inclusiva. Los resultados obtenidos por Musiclang en esta línea apuntan a que la mediación lingüística, trabajada a través materiales musicales, favorece el desarrollo de la empatía hacia los demás y su conciencia social. Explicando y prediciendo el comportamiento de otras personas, los estudiantes de idiomas pueden alcanzar mayores niveles de competencia socioemocional y reducir las brechas interculturales y las desigualdades sociales (Cores-Bilbao, Fernández-Corbacho, Machancoses \& Fonseca-Mora, 2019).

Paralelamente, el tercer núcleo temático de Musiclang se ha centrado en el estudio del videoclip musical como artefacto cultural (Martos \& García, 2014) de mayor consumo entre los jóvenes europeos. Considerando la cultura audiovisual, el desarrollo del pensamiento crítico y la alfabetización mediática como elementos esenciales para el currículo postmoderno (Triviño Cabrera, 2017), así como la interrelación existente entre las preferencias en el consumo de medios audiovisuales y la percepción intercultural del alumnado universitario (Godwin-Jones, 2015), el proyecto apuesta por exponer al alumnado no solo a la lectoescritura convencional sino a la oralidad, la cultura mediática y la cibercultura. En este sentido, el proyecto identifica la repercusión de los productos mediáticos con mayor número de visualizaciones sobre la identidad sociocultural y sobre diferentes aspectos de la comunicación intercultural del alumnado (Sánchez-Vizcaíno \& Fonseca-Mora, 2019), además de aportar propuestas para la funcionalización pedagógica (Sánchez Villarroel, 2006) de los videoclips musicales.

La intersección de estas tres líneas temáticas da como resultado una novedosa propuesta metodológica para el desarrollo de la competencia lectora en lenguas extranjeras para adultos, que contempla la intervención de los factores rítmicos y melódicos para la comprensión y articulación del discurso, así como de mecanismos afectivos y elementos multiculturales, permitiendo un abordaje multidimensional del aprendizaje basado en recursos mediáticos. En este sentido, actualmente el equipo de investigación se halla inmerso en el diseño de un recurso multimodal interactivo en el que se compendiarán aquellos videoclips musicales cuyo contenido audiovisual y lingüístico propicien los valores interculturales entre la lengua materna y la lengua meta de alumnado, además de incidir en sus valores identitarios europeos.

\section{Referencias}

\section{Producción de Musiclang}

Cores-Bilbao, E., Fernández-Corbacho, A., Machancoses, F.H., \& Fonseca-Mora, M.C. (2019). A Music-Mediated Language Learning Experience: Students’ Awareness of Their Socio-Emotional Skills. Frontiers in Psychology, pp. 2238.

Cores-Bilbao, E., Méndez García, M.C., \& Fonseca-Mora, M.C. (2020). University students’ representations of Europe and self-identification as Europeans: a synthesis of qualitative evidence for future policy formulation. European Journal of Futures Research. 
Foncubierta, J. M. \& Fonseca-Mora, M. C. (2018). Comprender el proceso lector en segundas lenguas:cognición y afectividad. Revista Tejuelo, 28, pp.11-42.

Foncubierta, J. M., Machancoses, F. H., \& Fonseca-Mora, M. C. (2018). La competencia lectora del alumnado universitario en contexto AICLE. Porta Linguarum, Monograph (abril 2018), 75-88.

Fonseca-Mora, M.C. \& Fernández-Corbacho, A. (2017). Procesamiento fonológico y aprendizaje de la lectura en lengua extranjera. Revista Española de Lingüística Aplicada/Spanish Journal of Applied Linguistics (RESLA/SJAL), 30(1), p. 166 187.

Gomez-Dominguez, M., Fonseca-Mora, M. C., \& Machancoses, F. H. (2019). First and foreign language early reading abilities: The influence of musical perception. Psychologyof Music, 47(2), 213-224.

Legaz, H., (2018). Fluidez lectora silenciosa, aptitud musical y lengua extranjera. Revista Tejuelo, 28, pp.161-184.

Méndez García, M.C., Cores-Bilbao, E., \& Moreno Gámez, L. (2019). Andalusian university students' perception of their European identity: international orientation and experiences. Journal of Multilingual and Multicultural Development.

Sánchez-Vizcaíno, M. C., \& Fonseca-Mora, M. C. (2019). Videoclip y emociones en el aprendizaje del Español como Lengua Extranjera. Círculo de Lingüística Aplicada a la Comunicación, pp. 255 - 286.

\section{Otras referencias}

Godwin-Jones, R. (2015). Emerging technologies the evolving roles of language teachers: trained coders, local researchers, global citizens. Language, Learning and Technology, 19(1), 10-22.

Habib, M., Lardy, C., Desiles, T., Commeiras, C., Chobert, J., \& Besson, M. (2016). Music and dyslexia: a new musical training method to improve reading and related disorders. Frontiers in Psychology, 7, 26.

Karma, K. (2007). Musical aptitude definition and measure validation: Ecological validity can endanger the construct validity of musical aptitude tests. Psychomusicology: A Journal of Research in Music Cognition, 19(2), 79-90.

Martos, E., \& García, A. E. M. (2014). Artefactos culturales y alfabetización en la era digital: discusiones conceptuales y praxis educativa. Teoría de la educación. Revista Interuniversitaria, 26 (1 (en-jun)), 119-135. 
Moreno, S., Marques, C., Santos, A., Santos, M., Castro, S. L., \& Besson, M. (2008). Musical training influences linguistic abilities in 8-year-old children: more evidence for brain plasticity. Cerebral Cortex, 19 (3), 712-723.

Sánchez Villarroel, J. (2017). Análisis y funcionalización pedagógica de productos mediáticos: la publicidad. Revista Electrónica Diálogos Educativos, 6(11), 128-147.

Toscano-Fuentes, C. M. (2010). Estudio empírico de la relación existente entre el nivel de adquisición de una segunda lengua, la capacidad auditiva y la inteligencia musical del alumnado. Tesis doctoral. Universidad de Huelva

Triviño Cabrera, L. (2017). Cultura audiovisual, alfabetización (literacidad) mediática y performance para la formación del profesorado en educación ciudadana. http://hdl.handle.net/10630/13866 